What inferences must we draw from these facts. Mosquitoes may carry the plasmodium malariæ from the blood of a patient afflicted with malaria to one who is not, as flies may carry the tubercle bacilli from the sputum of a tuberculous patient to other persons and deposit it on or about them; they will not all become infected, only a very small percentage of them. Mosquitoes can not develop malaria de novo. Persons who are perfectly well may go into a malarial district in the early spring-before mosquitoes are hatched and developed-and work in the ground and contract malaria without ever seeing a mosquito. Foster defines malaria as "air tainted by deleterious emanations from animal or vegetable matter, especially noxious exhalations of marsiny districts, capable of causing fever or other disease." This definition is in strict accord with all of our exact knowledge of malaria.

Dr. O. T. Osborne, New Haven, Conn.-I should like to emphasize Dr. Davis' point. I have had somewhat the same experience in New Haven that he has had in Chicago. Fifteen years ago there were a great many cases of intermittent fever. From that time until five years ago we had very little. In the center of the city there was almost none. Then they began to put up a number of large buildings, dug cellars, put in electric cars, which went all over the city; the streets were much dug up. From that time typical intermittent fever reappeared. New Haven is accustomed to a few mosquitoes; we have very little trouble with them and seldom do we find it necessary to screen against them.

Dr. R. C. Newton, Montclair, N. J.-I should like to ask if it is true, as has been frequently asserted, that malaria does not originate in localities which are over 700 feet above the sea-level. And if this popular belief is well founded, what shall we say about the presence of the anopheles? Is it found at an altitude of 700 feet or over above the sea-level?

Dr. W. S. Thayer, Baltimore-With regard to the effect of the mosquito bite one should remember that it is not the number of mosquitoes which bite one, but the kind of mosquito, and the result depends entirely on whether or not it is infected. The great mass of mosquitoes all over the country are perfectly harmless, as far as malaria goes. There is only one genus of mosquito which is capable of transferring the disease and this genus inhabits those regions which we know as malarious. Members of this genus are, however, present in other regions which may not generally be known as malarious but which may be shown to be potentially so, as was the case in the epidemic which has recently been referred to, following the advent of Italian laborers. And in such districts when one looks carefully into the history of the region he will probably find that malaria has, at one time or another, existed. There is really no evidence to show that malaria can be acquired through the gastro-intestinal tract. There is simply no positive evidence that any epidemic of malaria is due to an infected water-supply.

Dr. L. O. Howars, U. S. Department of Agriculture-In answer to the question asked regarding the occurrence of malarial mosquitoes at an altitude above 700 feet I would state that they have been observed at an elevation of 1000 feet and over.

Dr. Albert Woldert, closing the discussion-Experiments have shown that the length of time the anopheles remains infected after it has bitten a case of malarial fever is about three weeks. After this length of time has elapsed it is believed the insect ean not inoculate man with malarial fever.

Referring to the subject of malarial hemoglobinuria, in my opinion the etiology is not definitely known. Many cases of hematuria of doubtful origin have been spoken of as malarial hemoglobinuria. A distinction should be made between hemoglobinuria and hematuria by the use of the microscope. At one hospital I know of, over 2000 ounces of quinin had been given to patients in which the elinical diagnosis of malarial fever had been made, without producing hemoglobinuria. Of five cases of supposed malarial hemoglobinuria all took quinin and all recovered.

Dr. William Britr. Burss, Deckerville, Ark.-I came to this meeting to especially make a plea for harmony in the treat- ment of hemoglobinuria. As my reports show-of course, 16 cases does not show a large clinical report-more than onehalf the cases died under the treatment, without quinin. It is the custom for many physicians in the southern states to use no quinin in the treatment of hemoglobinuria. One year I was losing all my cases and I determined to try one case, and see if he would live if I used quinin. This patient had considerable jaundice and an enlarged spleen, a typical case, in short, wherein those, who fear quinin, would have withheld that agent. He had been given quinin sulphate in large doses by the mouth, and on the third day developed hemoglobinuria. How easy it would have been to have arrived at a quinin-hemoglobinuria. Quinin saved that man's life, I believe; $X$ began at once, not to withdraw, but to get quinin into the circulation, giving $7 \frac{1}{2}$ grain doses every four hours of the hydrochlorate, intra-gluteally, and to my surprise he got well; moreover this same person was treated successfully with quinin in a second attack some months later. Ever since, $I$ have treated every case with calomel, turpentin, hot applications and beef-juice I have also been using the normal salt solution, per rectum, which I find replenishes the blood and allays thirst. This mode of treatment, with quinin applied, in the past five cases has proved successful. I object to the term post-malaria in any form; I know of no post-malarial condition, except con. valescence and sound health. I find estivo-autumnal parasites in all cases of hemoglobinuria, and knowing the great hemolysis wrought by these agents, I would doubt the wisdom of withholding the one agent which would eliminate the plas modia from the blood. I no longer fear quinin in hemoglobinuria.

\section{MASSAGE OF THE EYEBALL.}

PRESENT STATUS AND VALUE, WITH THE CONSIDERATION OF WHAT DISEASES OF THE EYEBALL MAY BE FAVORABLY INFLUENCED BY THIS THERAPEUTIC II EASURE AND WHAT ARE THE BEST MEANS OF ITS APPLICATION.* CASEY A. WOOD, M.D. CHICAGo.

Massage is one of the oldest remedies known to ophthalmic surgery, and it may well be believed that a method of treatment not only in vogue thousands of years ago, but also adopted and retained by almost every school of medicine, must have special virtue.

Having used ocular massage extensively for many years, I gladly avail myself of this opportunity of exchanging with my colleagues opinions as to its efficacy.

First, I wish to speak particularly of the simple and not of the instrumental variety, such as the direct rubbing with pieces of cotton wool or some fabric mounted on a convenient shaft, the tapottement (or tetanization) of Maklakow, the use of sounds, with or without buttonheads, and other devices. In the main, indirect massage (Pagenstecher, Michel) with the pulp of the finger placed on the skin of the lids is to be preferred. Costomiris, formerly of Athens, who speaks enthusiastically of massage, believes that the best results are obtained by the direct rubbing of the finger tip or tips on the exposed cornea and conjunctiva. "So far as the latter membrane is concerned, I believe direct massage is valuable, and in some instances decidedly preferable to the indirect method, but the stroking movements, with slight pressure on the exterior of the eyelids, are usually sufficiently efficacious, are certainly less difficult to carry out in practice, and are more readily borne by the majority of American patients.

It is necessary to say that the amount of force used, the length of cach sitting, the frequency of the appli-

* Presented to the Section on Ophthalmology, at the Fifty-first Annual Meeting of the American Medical Association, held at Annual Meeting of the American
Atlantic City, N. J., June 5-8, 1900 . 
cations, the strength and character of the remedial agent-if any-used with the rubbing, and the susceptibility of the patient, are prime factors in the successful employment of massage, and are quite as important as the selection of cases appropriate for this remedy. Let us consider these in the order stated.

In indirect massage the act should never of itself produce pain, and rarely more than a passing discomfort. I think little is to be gained by the employment of rimch force. If the lids are the parts to be treated, the paitient siould be told to look down in massaging the upper lid, and up in the case of the lower lid. In each instance, the other lid should be drawn away from the one undergoing massage. If the cornea is to receive attention, the patient should look straight forward. The duration of the séance must be varied according to circumstances; it should rarely exceed three or four minutes. The tip of the second finger I have found the best instrument for the application, as it can readily be used for all the movements of palpebral massage, circular, centrifugal, and centripetal. It is also well adapted to the to-and-fro pressure required to reach the conjunctival areas about the inner and outer canthi. Once daily to twice a week constitutes the range of frequency of these applications. It is better in most cases to perform a gentle, non-irritating massage of even ten minutes' duration daily than a rough, painful rubbing twice a week.

As to the strength and character of remedial adjuncts, and without discussing the action of massage, which forms no part of this paper, I have come to rely on variations of some half dozen remedies. When I desire to use massage for its own sake, as I usually do, I instill a drop or two of cod-liver or pure castor-oil, telling the patient to wink and move the globe about so as to thoroughly distribute the oil throughout the conjunciival sac before beginning massage. For the rest I greatly prefer oily solutions or mixtures to powders or collyria. When these oleaginous compounds are made perfectly smooth and of a consistency that permits of their ready distribution over the eyeball, it is surprising how little pain or discomfort is set up even by strong doses of such irritants as mercuric chlorid, silver nitrate, etc. This is probably due to the fact that massage has an anesthetic action, probably due to emptying the capillaries and lymph-vessels of their contents and to the continued pressure on the nerve endings.

Most observers find in mercurials of various strengths, combined with all sorts of oleaginous excipients, the most useful massage agents, and my experience coincides with theirs. I pass around for your inspection a brown ointment of this character that $I$ have found very effective, and one that combines qualities of great value in ocular massage. It is nothing but the old citrine ointment-the ointment of the nitrate of mercury-made with brown cod-liver oil instead of lardoil. This, after standing exposed, to get rid of irritating nitrous fumes, for a week or ten days to the air, but not to dust, may be further diluted with from 25 to 50 per cent. of cod-liver oil. It should be dropped from the end of a glass rod or silver probe into the lower sac. If the massage movements are begun at once, the smarting is very slight and transient, even when the stronger mixtures are employed. The subsequent action of the remedy can be modified as desired by the length of time, usually an hour, before it is washed off the evelashes with warm watcr.

At the end of, or during the act, combinations of the remedy with the ocular secretions-especially mucus- should be coaxed out of the sac by small "dabs" of damp cotton, or, if watery or glycerin mixtures or solutions be employed, by the irrigating stream, and the stroking movements resumed until nothing further comes away.

I wish to emphasize the contention that one rarely gains anything by inducing a marked hyperemia of the scleral or ciliary vessels by using force or by the employment of very irritating adjuncts in massage. A little smarting, that passes off in five minutes; a temporary congestion of the already visible vessels, and, perhaps, some foreign body sensations are all that are justifiable. I might generalize by saying that the patient, half an hour after the lid friction, should not experience any added discomfort. It is evidently impossible for me to even enumerate the various diseases of the lid proper, conjunctiva, lachrymal apparatus, cornea, and of the interior of the eye, in whose treatment massage has many warm friends. In a general way its most satisfactory employment will be found in chronic diseases of the eye borders and substance, in almost all those subacute and chronic infections of the conjunctivæ that one commonly includes in the title "conjunctivitis" - with or without an adjectival affix or prefix-in the second stage of acute inflammation of the conjunctiva, in most forms of ulcer of and deposit in the cornea, and it may be employed for the temporary relief of glaucoma and in some forms of retinal embolism. It is not indicated in the early stages of "acute conjunctivitis" and of "keratitis," in all forms of irue trachoma, in spring catarrh-although Costomiris and others dissent from this opinion-in diseases of the iris, ciliary body, lens, choroid, vitreous, or optic nerve. Finally, while I am very skeptical of its efficacy in the absorption of the connective tissue that composes the mass of a true cicatrix, I am convinced that, in young subjects, especially, it does lessen the opacity following ulcer of the cornea. If applied early it promotes the removal of the surrounding infiltrate that would otherwise remain. In the same way it is valuable in the treatment of interstitial keratitis.

\section{USE AND ABUSE OF POTASSIUM IODID IN OPHTHALMIC PRACTICE.*}

\section{ALBERT RUFUS BAKER, M.D.}

Professor of Diseases of the Eye, Far and Throat in the Cleveland College of Physicians and Surgeons; Oculist to the Cleveland General. City, and Saint Alexis Hospitals. CLEVELAND, OHIO.

Iodid of potash was first used medicinally by veterinary surgeons for the removal of splints from horses. Its efficacy in removing these osseous growths suggested its use for the removal of tumors and exudates from man. Its value in the cure of many tertiary syphilitic lesions soon became apparent, and it is slowly but surely forcing the medical profession to acknowledge that its use is not restricted to syphilitic diseases.

Many years ago I became convinced of its value in the removal of goiter. I recall a case many years since that forcibly impressed on my mind the efficacy of iodid of potash in cases other than syphilitic ones. I was in attendance on a little girl with pneumonia, but resolution failed to become established. The lung remained solid several weeks. The child was slowly but surely dying. The father, a physician, as well as myself folt helpless, and we called in council an old prac-

* Presented to the Section on Ophthalmology, at the Fifty-first Annual Meeting of the American Medical Association, held at Atlantic City, N. J., June 5-8, 1900. 